\title{
Bubble-Like Response of Living Blood Cells and Microparticles in an Ultrasound Field
}

\author{
N. MAzzAwi ${ }^{a}$, M. POSTema $^{b, c, *}$ And E. Kimmel ${ }^{a}$ \\ ${ }^{a}$ Department of Biomedical Engineering, Technion, Haifa, Israel \\ ${ }^{b}$ Department of Physics and Technology, University of Bergen, Bergen, Norway \\ ${ }^{c}$ The Michelsen Centre for Industrial Measurement Science and Technology, Bergen, Norway
}

\begin{abstract}
The bilayer sonophore model suggests that ultrasound induces a pulsating structure in the intra-membrane hydrophobic space between the two lipid monolayer leaflets of the cell membrane, assembled by dissolved gas of the surrounding area, which absorbs acoustic energy and transforms it by creating intra-cellular structural changes. This void has been referred to as a bilayer sonophore. The bilayer sonophore inflates and deflates periodically when exposed to ultrasound and may itself radiate acoustic pressure pulses in the surrounding medium in the same way a gas bubble does: once exposed to ultrasound the bilayer sonophore becomes a mechanical oscillator and a source of intracellular cavitation activity. In this paper, we describe observations of the clustering behaviour of living cells and several other particles in a standing sound field generated inside a ring transducer. Upon sonication, blood cells and monodisperse polystyrene particles were observed to have been trapped in the same locations, corresponding to nodes of the ultrasound field. Because polystyrene is hydrophobic, it behaves like a particle trapped inside a thin gas shell. In fact, the sonophore model treats biological cells in a similar way. Microbubbles that form the ultrasound contrast agent Quantison ${ }^{T M}$ behave differently, however. These microbubbles accumulated in circles faster than blood cells and polystyrene particles. In addition, they form tightly packed clusters at the nodes, indicating very strong secondary Bjerknes forces. Cluster formation is not to be expected in cells with predicted sonophore sizes on the order of 10-100 nm.
\end{abstract}

DOI: $10.12693 /$ APhysPolA.127.103

PACS: $43.80 . \mathrm{Cs}, 47.55 . \mathrm{df}, 87.80 . \mathrm{Fe}$

\section{Introduction}

The bilayer sonophore model suggests that ultrasound induces a pulsating structure in the intra-membrane hydrophobic space between the two lipid monolayer leaflets of the cell membrane, assembled by dissolved gas of the surrounding area, which absorbs acoustic energy and transforms it by creating intra-cellular structural changes [1]. The two leaflets are pulled apart when the

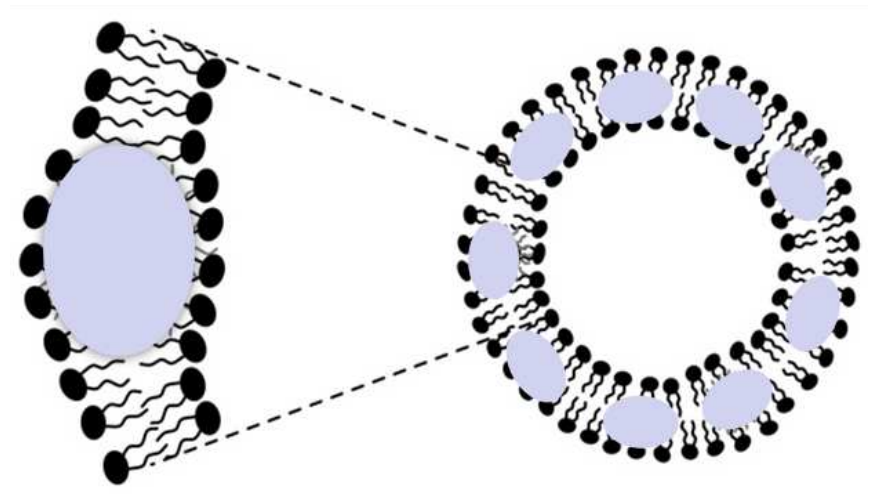

Fig. 1. Bilayer sonophore formation within the intramembrane space of a cell.

${ }^{*}$ corresponding author; e-mail: michiel.postema@ift.uib.no acoustic rarefaction pressure overcomes the molecular attractive forces between them and are pushed back together by the compressive pressure. This void has been referred to as a bilayer sonophore (cf. Fig. 1). The bilayer sonophore inflates and deflates periodically when exposed to ultrasound and may itself radiate acoustic pressure pulses in the surrounding medium in the same way a gas bubble does: once exposed to ultrasound the bilayer sonophore becomes a mechanical oscillator and a source of intracellular cavitation activity.

In this paper, we describe observations of the clustering behaviour of living cells and several other particles in a standing sound field.

\section{Methods}

Figure 2 shows a schematic overview of the experimental setup used in this study. The main element of the setup is a ring transducer consisting of a Pz26 (OD32 ID28 L14) piezoceramic tube of lead zirconate titanate (PZT) (Ferroperm Piezoceramics, Kvistgård, Denmark) with an inner diameter of $28 \mathrm{~mm}$, a height of $10 \mathrm{~mm}$, and an element thickness of $2.1 \mathrm{~mm}$. The ring transducer was fixed to the centre of a Petri dish with a $100 \mathrm{~mm}$ diameter and a $15 \mathrm{~mm}$ height. The Petri dish was filled with a $2 \%$ Agar solution. The transducer was connected to an AR75 power amplifier (AR Company, Souderton, PA, USA), which was connected to either a 8024 arbitrary waveform generator (Tabor Electronics, Irvine, CA, USA) or a 33120A arbitrary waveform generator (Hewlett-Packard 
Company, Palo Alto, CA, USA). The signal generated consisted of a continuous $1 \mathrm{MHz}$ or $3 \mathrm{MHz}$ sine wave with peak-to-peak amplitudes between $1 \mathrm{~V}$ and $10 \mathrm{~V}$.

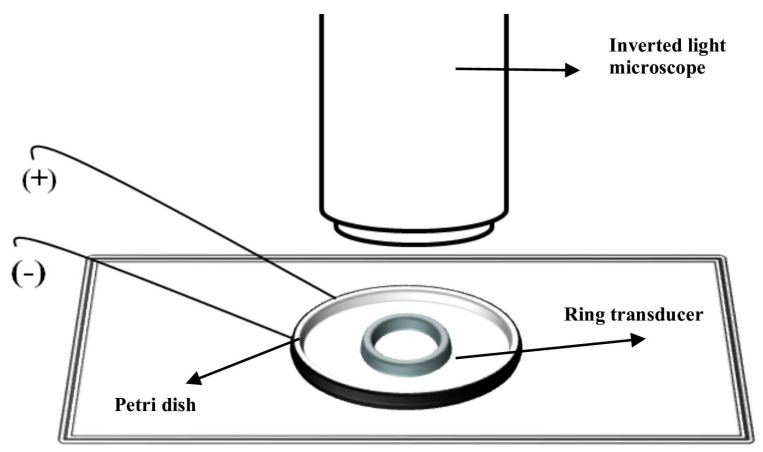

Fig. 2. Schematic of experimental setup.

The centre of the Petri dish was mounted under an Eclipse TE2000 inverted microscope (Nikon Instruments Inc., Melville, NY, USA) with $2 \times, 10 \times$, and $20 \times$ magnification, to which an Infinity $3-1 \mathrm{M}$ camera (Lumenera Corp., Ottawa, Ontario, Canada) was connected.

Among the objects studied were (a) $0.1 \mathrm{ml}$ of rat blood mixed with $0.02 \mathrm{~g}$ of heparin dissolved in a $2 \mathrm{ml} \mathrm{PBS}$ solution added to $2 \mathrm{ml}$ of DMEM and $0.1 \mathrm{ml}$ of trypsin, (b) Duke Standards 4210A, 4220A polystyrene microspheres (Thermo Fisher Scientific Inc, Waltham, MA, USA), (c) Quantison ${ }^{T M}$ ultrasound contrast agent (Upperton Limited, Nottingham, UK).

\section{Results and discussion}

Upon sonication, particles and cells translated towards nodes of the sound field. As sonication stopped, all trapped objects maintained at these positions, whilst other free (non-trapped) objects have gradually stopped moving and circulating inside the ring. The location of the nodes and antinodes of the sound field was dependent upon the transmit frequency used.

Blood cells that had been trapped at the nodes were arranged in circles inside the ring, as shown in Fig. 3 . The average radial distance measured between these circleshaped strips was half a wavelength. This finding indicated that the resonance frequency of all cells is below the transmit frequency. In a previous study with an ultrasound contrast agent with a wide size distribution, the space between microbubble clusters was deducted and found to be a quarter wavelength [2], because the greater microbubbles would be trapped in the nodes and the smaller microbubbles would be trapped in the antinodes of the sound field.

Upon sonication, the monodisperse polystyrene particles were observed to have been trapped in the same locations as the blood cells. Because polystyrene is hydrophobic, it behaves like a particle trapped inside a thin gas shell. In fact, the sonophore model relates to biolog-

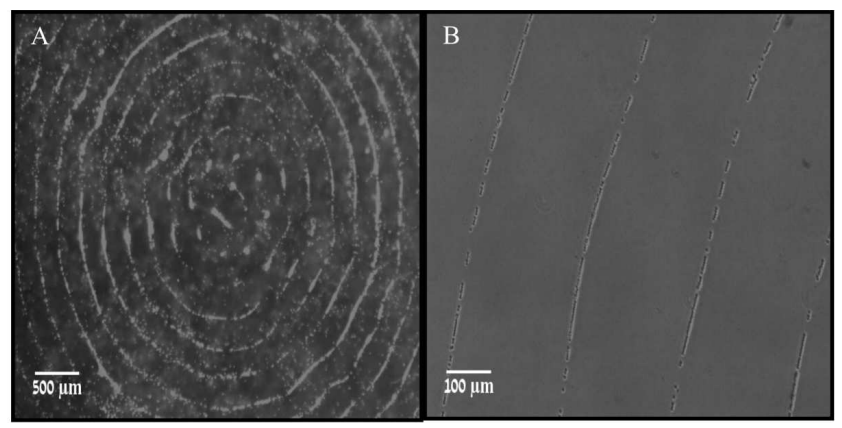

Fig. 3. Blood cells arranged in concentric circles inside the ring transducer after $3 \mathrm{MHz}$ sonication, observed at A) $2 \times$ magnification, and B) $10 \times$ magnification.

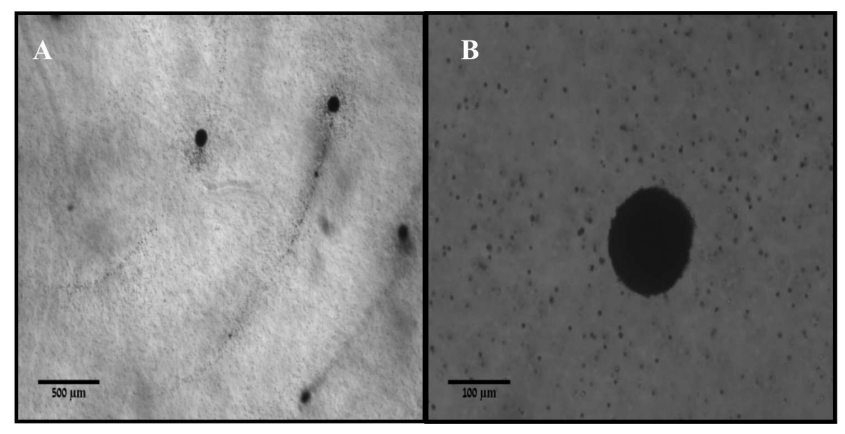

Fig. 4. Quantison ${ }^{\top M}$ microbubbles not only accumulate in circles, corresponding to the nodes of the $1 \mathrm{MHz}$ ultrasound sound field, but also form tightly packed clusters at the nodes, when observed at A) $2 \times$ magnification and B) $10 \times$ magnification.

ical cells in a similar way, except for the difference that sonophores develop only in a sound field.

The microbubbles of which the ultrasound contrast agent Quantison ${ }^{\mathrm{TM}}$ consists behave differently, however, as demonstrated in Fig. 4. These microbubbles with mean diameters of $5 \mu \mathrm{m}$ accumulated in circles faster than blood cells and polystyrene particles. Again, these circles correspond to the nodes of the ultrasound field. In addition, they form tightly packed clusters at the nodes, indicating very strong secondary Bjerknes forces [2].

Cluster formation is not to be expected in cells, because the sonophore sizes predicted from [1] are on the order of 10-100 nm. Given the fact that secondary Bjerknes forces of gas bubbles are proportional to the bubbles radius to the power of five [3], these forces must be too weak if created by sonophores. Moreover, cells in the nodes are subjected to zero acoustic pressure and therefore no sonophore formation is to be expected in standing waves [1].

In order to compare any bubble-like behaviour of cells with actual bubble-like systems, a comparison with antibubbles would be interesting, especially in a running sound field. Recently, it has been disclosed how to manufacture stable antibubbles, consisting of a liquid core, 
of the same dimensions as a blood cell, and a gaseous shell [4]. If the sonophore hypothesis is correct, blood cells must demonstrate the same acoustic properties as antibubbles.

\section{Conclusions}

Although blood cells and polystyrene particles accumulate slower than ultrasound contrast agent microbubbles, their acoustic behaviour has similarities. The absence of tightly packed cluster formation in cells indicates that the secondary Bjerknes forces are too low for this to happen, which corresponds to sonophore theory.

More studies, e.g., by comparing the acoustic behaviour of blood cells with that of antibubbles, will bring us closer to finding whether cells form sonophores upon sonication.

\section{Acknowledgments}

The scholarship of Nasma Mazzawi has been financed by the Technion. The ring transducer was kindly given to us by Dr. Graham Brodie from the University of Dundee.

\section{References}

[1] B. Krasovitski, V. Frenkel, S. Shoham, E. Kimmel, Proc. Natl. Acad. Sci. 108, 3258 (2011).

[2] S. Kotopoulis, M. Postema, Ultrasonics 50, 260 (2010).

[3] M. Postema, Fundamentals of Medical Ultrasonics, Spon Press, New York 2011.

[4] A.T. Poortinga, Langmuir 27, 2138 (2011). 